\title{
To Catch a Thief: Practical Methods of Using Social Networks as a Mechanism for Identifying Corporate Insider Threats
}

\author{
Martyn Styles \\ University of South Wales, United Kingdom \\ $03157210 @ g l a m \cdot a c \cdot u k$
}

\begin{abstract}
Is it possible to utilize psychological profiling through social network analysis to identify potential corporate insider threats? This research will aim to provide corporate information security teams with techniques capable of recognizing the signs of an insider threat.
\end{abstract}

Keywords: Cyber security, social networking, information security, crime.

\section{Introduction}

The insider threat to organizations is bigger than ever before, thanks in part to the growing trend amongst certain individuals who believe that terms such as "confidential", "non-disclosure" and "private" no longer apply to any piece of data which, for reasons of ethics, conscience or organization profit should normally remain safeguarded within the boundary of the office walls. This research will attempt to illustrate the possibilities for identifying corporate insider threats through analyzing social networks, with the aim of identifying internal individuals who are potential data security threats through keyword analysis of popular social and professional networks such as Facebook, Twitter and LinkedIn.

\section{Insider Definition}

An insider is generally defined as any person within an organization who accesses data which may be regarded as useful by external persons if extracted from the organization, without the full knowledge of that organization. The oxymoron of the 'insider threat' is such that information security teams find it challenging to even countenance the thought that the typical employee has the capability to 'go rogue'. Information security teams are generally primed to detect threats from the outside trying to get in, rather than identify employees trying to egress data out of the organization.

Following the precedent of trust-betrayal by highly privileged serial-leakers; such as Chelsea/Bradley Manning and Edward Snowden, corporate employees may be tempted to release business secrets for amusement or financial gain. Although recent research 
has been performed to help identify insider threats (Claycomb W.R. et al., 2011) (Greitzer and Hohimer, 2011) (Greitzer et al., 2010), very little has been accomplished by using social network data pertaining to an organization. A recent model for understanding insider threats was proposed by Legg P. et al. (2013), which builds on earlier work by Magklaras and Furnell (2002) and Anderson (1980) to help describe a system capable of identifying indicators of misuse in IT systems. Anderson (1980)describes three categories of internal abuse: 1. Masqueraders - insiders that exploit security weaknesses in systems 2. Misfeasors - an insider who abuses legitimate privileges 3 . Clandestine users - insiders with significant privileges that enable them to hide from audit logs. For the purposes of this research, we are looking for indications of a Misfeasor (in law, the abuse of lawful authority in order to achieve a desired result.) - a user of a system who exercises their legitimate access to data to remove it from the organization. Although Small (2009) says "If systems are compromised, does it matter if the cause was malice, misuse or mistake?", I believe that mistakes are a necessary risk in any business, however malice and misuse are avoidable.

Both masqueraders and clandestine users are typically I.T. employees, but the methods used to extract information from the organization are often similar to the misfeasor - typical egress mechanisms being through the use of email, web page uploads, file sharing sites or USB memory stick.

\section{$3 \quad$ Privacy and Ethical Issues}

Evidently there are a number of privacy and ethical issues raised with researching social networking data. In Europe, the EU Privacy Directive governs the rights of individuals for their personal data behavior to remain un-monitored, however in a corporate environment there are exceptions given a reasonable suspicion of wrongdoing and where the data movement between the individual and a social networking site involves corporate data. Ethical considerations for social media research will determine the extent to which investigations into end-user social networks can be exploited, using guidelines published by British Psychological Society (2010), Moreno et al. (2013), Henderson et al. (2012) and Zimmer (2010), and it is expected that for the purposes of the research paper, all personal details will be anonymized.

\section{Insider Detection Process Flow}

The process flow of insider detection is based on the following:

- Egress Detection: Detect unauthorized outgoing corporate data streams

- Social Net Analysis: Assess social network content related to the perpetrator

- Behavioral Profiling: Inaugurate behavioral profiling of the individual

- Threat Identification: Identify these activities as potential insider threat

Egress Detection: To initially aid in the identification of corporate data theft, we use commercial Data Leak Prevention (DLP) tools to flag emails with attachments 
containing potentially confidential data, sent from internal users to GoogleDocs, Gmail, Yahoo!Mail and other personal file sharing websites, since this method of data egress can be a strong indication of an insider threat. However useful this method is, using it to identify insiders may be limited because quite often false positives incorrectly trigger as DLP alerts through misguided users sending documents to home computers as a short-cut means of completing work or they are simply personal files such as photographs.

Social Net Analysis: Commercial tools such as IBM i2 Analyst's Notebook and Paterva Maltego facilitate the analysis of multiple social network streams in a visual manner. For this research project we are using Maltego and a selected number of online tools for social network analysis. To test the capability of using social networks to indicate potential insiders, a taxonomy of key words associated with the business activities carried out by an organization was refined for use in the social networking searches, and a list of current users was imported into Maltego to facilitate a search of social networks for keywords. The software has the ability to search a variety of different social networks as well as sites like pastebin.com, which is commonly used to leak information. With Maltego we search for interesting files found on social media sites that relate to the list of users.

Behavioral Profiling: Psychological profiling for potential insider threats is suggested by Shaw (2006), Stanton et al. (2005) and Crossler et al. (2013). When verifiable confidential corporate data is egressed through email attachments to personal email domains or file sharing website uploads, DLP alert data can be passed into Maltego and linked to the user's 'Person' item, so that a diagram can be formed of the relationships. In this way we can begin to understand confidential data movement beyond the corporate firewall.

Threat Identification: A combination of corporate data egress detection and social network analysis are used in the identification of insider threats. We use Maltego to visualize the insider threat.

\section{Conclusion}

The experiments we have undertaken thus far indicate that social network analysis is a valid method of detecting potential insider threats. Further work needs to be performed to validate our findings, however early indications are encouraging that the use of social network analysis is a useful mechanism for detection of an insider threat.

\section{References}

1. Anderson, J.P.: Computer security threat monitoring and surveillance. James P. Anderson Co. (1980)

2. British Psychological Society, Code of Ethics and Conduct (2010) 
3. Claycomb, W.R., Huth, C.L., Flynn, L., Mcintire, D.M., Todd, B.: Chronological Examination of Insider Threat Sabotage: Preliminary Observations. CERT Insider Threat Center, Carnegie Mellon (2011)

4. Crossler, R.E., Johnston, A.C., Lowry, P.B., Hu, Q., Warkentin, M., Baskerville, R.: Future directions for behavioral information security research. Computers \& Security 32, 90101 (2013)

5. Greitzer, F., Kangas, L., Noonan, C., Dalton, A.: Identifying at-Risk Employees: A Behavioral Model for Predicting Potential Insider Threats (2010)

6. Greitzer, F.L., Hohimer, R.E.: Modeling Human Behavior to Anticipate Insider Attacks. Journal of Strategic Security 4(2), Article 3 (2011)

7. Henderson, T., Hutton, L., Mcneilly, S.: Ethics and online social network research - developing best practices. In: BCS HCI Workshop on HCI Research in Sensitive Contexts: Ethical Considerations, Birmingham, UK (2012)

8. Legg, P., Moffat, N., Nurse, J.R.C., Happa, J., Agrafiotis, I., Goldsmith, M., Creese, S.: Towards a Conceptual Model and Reasoning Structure for Insider Threat Detection (2013)

9. Magklaras, G.B., Furnell, S.M.: Insider Threat Prediction Tool: Evaluating the probability of IT misuse. Computers \& Security 21, 62-73 (2002)

10. Moreno, M.A., Goniu, N., Moreno, P.S., Diekema, D.: Ethics of social media research: common concerns and practical considerations. Cyberpsychol Behav. Soc. Netw. 16, 708$713(2013)$

11. Shaw, E.D.: The role of behavioral research and profiling in malicious cyber insider investigations. Digital Investigation 3, 20-31 (2006)

12. Small, M.: The root of the problem - malice misuse or mistake. Computer Fraud \& Security, 6-9 (2009)

13. Stanton, J., Stam, K., Mastrangelo, P., Jolton, J.: Analysis of end user security behaviors. Computers \& Security 24, 124-133 (2005)

14. Zimmer, M.: "But the data is already public": on the ethics of research in Facebook. Ethics and Information Technology 12, 313-325 (2010)

\section{Social Networking Keyword Monitoring:}

1. http: / / backtweets. com/

2. http://google.com/alerts

3. http: / /www. icerocket. com/

4. http: / /www. netvibes. com

5. http: // tweetdeck. twitter.com/

6. http: / / www . paterva. com/web6/products /mal tego.php

7. http: / /www-03.ibm. com/software/products/en/ analysts-notebook/ 